\title{
SINGULAR PERTURBATION APPROACH TO AN ELASTIC DRY FRICTION PROBLEM WITH NON-MONOTONE COEFFICIENT
}

\author{
BY \\ YVES RENARD \\ LMC-IMAG, BP 53X, F-38041 Grenoble, France
}

\begin{abstract}
In this paper we study a one-dimensional dynamic model of dry friction with slip velocity dependent coefficient. In many cases, this model has more than one solution. We introduce a perturbed friction condition which allows us to regain the uniqueness of the solution. We show that the perturbed problem's solutions pointwise converge to a particular solution of the initial problem when the perturbation parameter tends to zero. The singular perturbation approach provides the analysis of a criterion used to select a solution of the problem, and suggests a method to study more elaborated dry friction problems.
\end{abstract}

1. Introduction. Models with dry friction and slip velocity dependent friction coefficient, especially when this coefficient has a decreasing part, were introduced to take into account stick-slip vibrations. Those vibrations are self-sustained oscillations induced by dry friction of elastic structures. They occur in engineering and geophysical systems as well as in everyday life. Because of the large number of factors which must be considered in dry friction processes, there does not exist a universally accepted model of this phenomenon. The simplest model, introduced by Euler, distinguishes between a static friction coefficient and a dynamic one. It was extended to a slip velocity dependent coefficient and studied by several authors on systems with a finite number of degrees of freedom [8], [9], [5], [12], with also an important application in the analysis of the sliding of geological faults [2], [1].

Concerning continuous models, very few studies have been made. Here we carry on the study of a one-dimensional model, which was initiated in [6] and [7]. In many cases, with such a friction condition, the dynamic system has many solutions. In spite of this, most of the redundant solutions are unsatisfactory from a mechanical point of view, in the sense that they do not correspond to an expected behavior of the system. The approach initiated by [6] and [7] in order to select the acceptable solution, is to introduce a criterion corresponding to the perfect delay criterion of catastrophe theory.

Received March 3, 1998.

2000 Mathematics Subject Classification. Primary 74M10, 74M15, 74A55.

(C) 2000 Brown University 
An important difference is that this criterion is applied here to a dynamic process even though it is normally used in the quasi-static framework.

In this paper we introduce a perturbed friction condition. Combining theories of multi-valued differential equations [3] and singular perturbation of differential equations [13], we prove that the one-dimensional problem with this perturbed condition has a unique solution which approaches a particular solution of the initial problem, i.e., that the sequence of solutions of the perturbed problem converges pointwise to a solution of the non-perturbed problem. The perfect delay criterion remains qualitative and only makes sense with sufficiently smooth solutions. We show that when this criterion selects a unique solution then this is the same as the solution that is approached by the perturbed problem. This study sheds a new light on the use of the perfect delay criterion in such dynamical problems. Moreover, such a perturbation approach is likely to be effective in the analysis of multi-dimensional friction problems (see [10]).

2. Dynamic problem with dry friction. The problem we deal with, because of its simplicity, allows us to study important properties of elastic systems under dry friction with slip velocity dependent coefficient. This model is composed of a homogeneous linear elastic slab, of height $H$, fixed on its top, which slides with dry friction on a rigid foundation in uniform motion with a horizontal velocity $V_{e}$ along the $x$-axis (see Fig. 1).

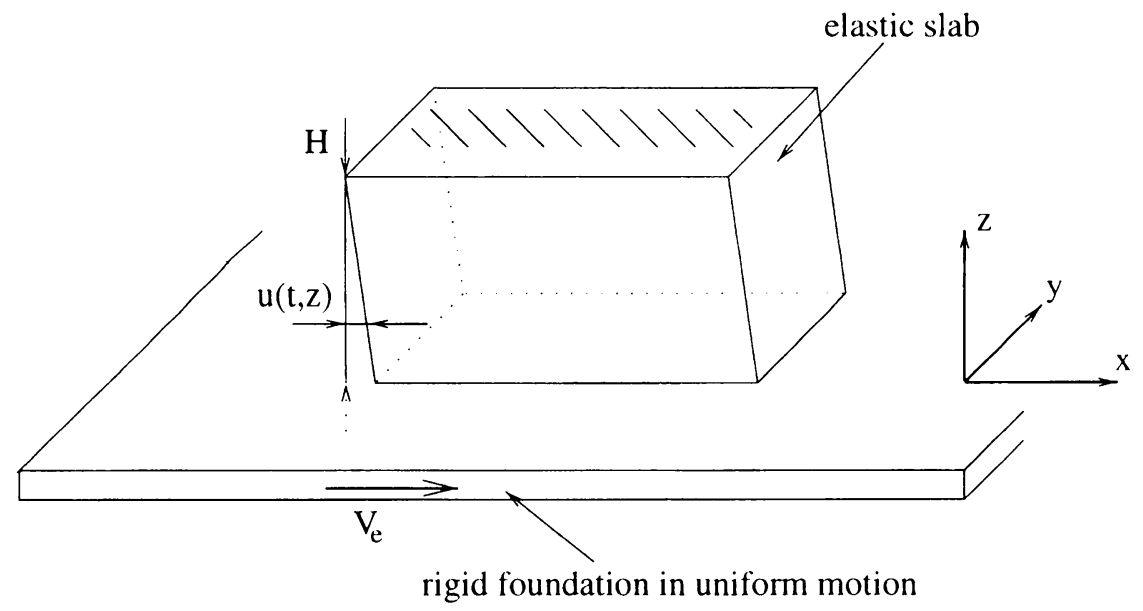

FIG. 1. Elastic slab sliding, with dry friction, on a rigid foundation that is in uniform motion $V_{e}$ along the $x$-axis

If we only consider the solutions that are constant along the horizontal coordinates, the displacement $u(t, z)$ along the $x$-axis is given by the following system of equations:

$$
\begin{array}{r}
\left.\left.\partial_{t t}^{2} u(t, z)-c^{2} \partial_{z z}^{2} u(t, z)=0, \quad z \in\right] 0, H[, \quad t \in] 0, T\right], \\
\left.\left.u(t, H)=0, \quad G \partial_{z} u(t, 0)=-F_{f}, \quad t \in\right] 0, T\right],
\end{array}
$$

where $F_{f}$ is the friction force, $G$ the shear modulus, $c=\sqrt{G / \rho}$ the shear wave velocity and $\rho$ the density. The friction force is given by a Coulomb law with slip velocity 
dependent coefficient:

$$
F_{f} \in-S \mu\left(\left|v_{s}\right|\right) \operatorname{Sgn}\left(v_{s}\right),
$$

where $\mu: \mathbb{R}^{+} \longrightarrow \mathbb{R}^{+}$is the friction coefficient, $S$ the contact pressure that is assumed to be constant, $v_{s}=\partial_{t} u(t, 0)-V_{e}$ the slip velocity on the contact boundary and Sgn the multi-valued sign function defined by

$$
\operatorname{Sgn}(x)= \begin{cases}{[-1,1]} & \text { if } x=0 \\ \left\{\frac{x}{|x|}\right\} & \text { if } x \neq 0\end{cases}
$$

The problem is completed by the initial conditions:

$$
u(0, z)=u_{0}(z), \quad \partial_{t} u(0, z)=u_{1}(z), \quad z \in[0, H] .
$$

Following Ionescu and Paumier [6] or Leonov and Srinivasan [7], and with the relation on characteristic lines for $t \leq H / c$ :

$$
\partial_{t} u(t, 0)+c \partial_{z} u(t, 0)=\partial_{t} u(0, c t)+c \partial_{z} u(0, c t),
$$

the problem (1)-(4) can be rewritten as a scalar inclusion on the contact boundary:

$$
\alpha(t) \in \beta\left(v_{s}(t)\right),
$$

with $\alpha$ and $\beta$ given by

$$
\alpha(t)=\frac{G}{c}\left(u_{1}(c t)-V_{e}(t)\right)+G \partial_{z} u_{0}(c t), \quad \beta(v)=\frac{G}{c} v+S \mu(|v|) \operatorname{Sgn}(v) .
$$

For any coefficient $\mu$, the map $\beta$ is not necessarily strictly monotone, and consequently does not always have an inverse function.

REMARK. The inclusion (6) corresponds to the contact problem only for $0 \leq t \leq H / c$. This is not an important limitation, because if the problem is solved for $0 \leq t \leq H / c$, then the same analysis can be developed for $H / c \leq t \leq 2 \mathrm{H} / \mathrm{c}$ with $t=H / c$ taken as the initial time. In the following, we consider $T \leq H / c$.

3. Nonuniqueness and perfect delay criterion. Let us take an example where $\mu$ reads:

$$
\mu(v)=\mu_{d}+\left(\mu_{s}-\mu_{d}\right) e^{-\frac{v}{v_{\mathrm{cr}}}},
$$

with $\mu_{s}>\mu_{d}$ ( $\mu_{s}$ as static coefficient and $\mu_{d}$ as dynamic coefficient), and $V_{\mathrm{cr}}>\left(\mu_{s}-\mu_{d}\right)$. This is a classical form for a slip velocity dependent coefficient, which is often used in practical models. Then the map $\beta$ is given by :

$$
\beta(v)= \begin{cases}\{v-S \mu(-v)\} & \text { if } \quad v<0 \\ {\left[-S \mu_{s},+S \mu_{s}\right]} & \text { if } \quad v=0 \\ \{v+S \mu(v)\} & \text { if } \quad v>0\end{cases}
$$

With $S$ sufficiently large, $\beta$ has the form shown in Fig. 2 and is not strictly increasing. Basic properties of $\beta$ (in particular, $\lim _{v \rightarrow \pm \infty} \beta(v)= \pm \infty$ ) make it possible for inclusion (6) to always have at least one solution $v_{s}$ for every function $\alpha:[0, T] \longrightarrow \mathbb{R}$. But generally, even if the function $\alpha$ is continuous, there are many solutions and all of them 


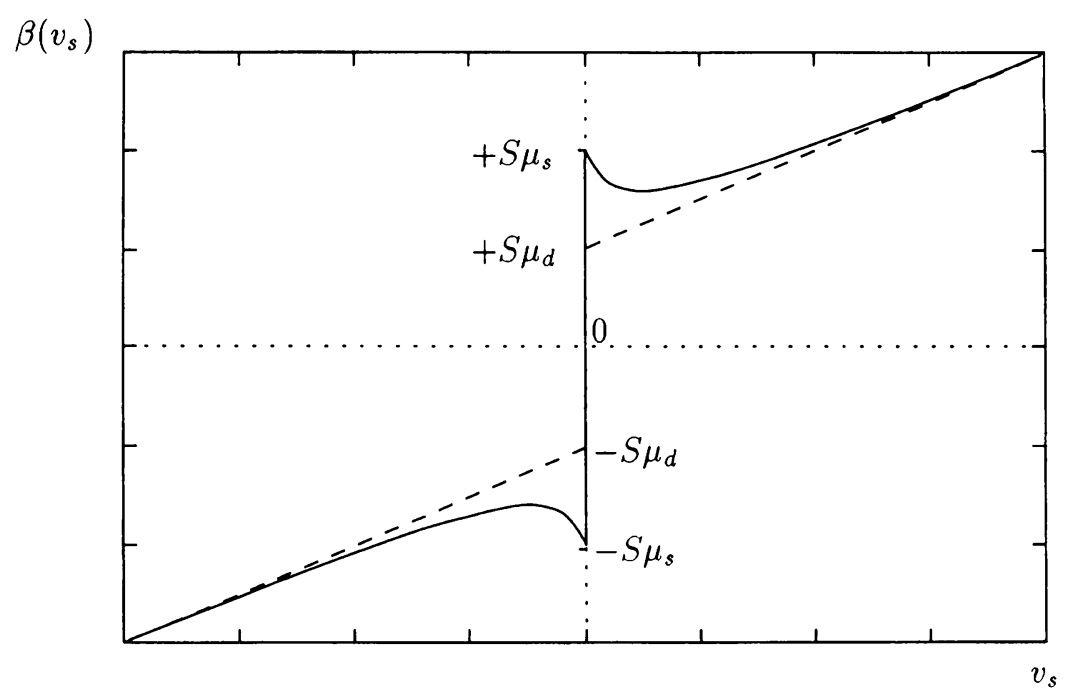

FIG. 2. The map $\beta$

are discontinuous. This is the case if, for instance, $\alpha$ is increasing from the value 0 to the value $2 S \mu_{s}$ (see Fig. 2).

When inclusion (6) has many solutions, some of them do not correspond to the mechanical behavior we expect from the system. For instance, still with $\alpha$ increasing from the value 0 to the value $2 S \mu_{s}$, we expect the system not to start sliding before the friction force reaches the value $S \mu_{s}$. For this reason, Ionescu and Paumier gave in [6] the following criterion in order to select the mechanically acceptable solution:

the system only jumps when it has no other choice.

Independently, Leonov and Srinivasan gave in [7] a criterion thatcan be written as:

at each instant $t$, we select the solution which minimizes $\left|v_{s}^{-}(t)-v_{s}^{+}(t)\right|{ }^{1}$

The second formulation gives supplementary information on which jump to choose. This defines a criterion, called the perfect delay criterion in catastrophe theory, which is normally used in the framework of quasi-static problems.

This analysis gives important information on the behavior of elastic structures under dry friction when a non-monotone coefficient of friction is used. The solutions of equations (1) and (2) can be seen as homogeneous motions of a two- or three-dimensional elastic structure sliding over a rigid foundation (see [10]). The previous analysis implies that, generally, the solution is not unique for purely elastic dynamic models, and also that the solutions are very irregular, with the occurrence of velocity shocks.

4. Perturbed friction condition. The aim of this paper is to introduce a perturbed friction condition which allows us to regain the uniqueness of the solution. Instead of

1 We denote respectively by $\alpha^{+}(t)=\lim _{\tau \rightarrow t^{+}} \alpha(\tau)$ and $\alpha^{-}(t)=\lim _{\tau \rightarrow t^{-}} \alpha(\tau)$ the right and left limits. 
the normal friction condition, which reads:

$$
G \partial_{z} u(t, 0) \in S \mu\left(\left|v_{s}(t)\right|\right) \operatorname{Sgn}\left(v_{s}(t)\right),
$$

we consider the following perturbed friction condition:

$$
\varepsilon \frac{d}{d t} v_{s}(t)-G \partial_{z} u(t, 0) \in-S \mu\left(\left|v_{s}(t)\right|\right) \operatorname{Sgn}\left(v_{s}(t)\right),
$$

where $\varepsilon$ can be interpreted as the (small) density of the contact surface. The elastic friction problem with such a perturbed condition can be rewritten as follows on the contact boundary thanks to (5):

$$
\varepsilon \frac{d}{d t} v_{s}(t) \in \alpha(t)-\beta\left(v_{s}(t)\right)
$$

with $\alpha$ and $\beta$ still given by (7). This is a singular perturbation of inclusion (6). It is
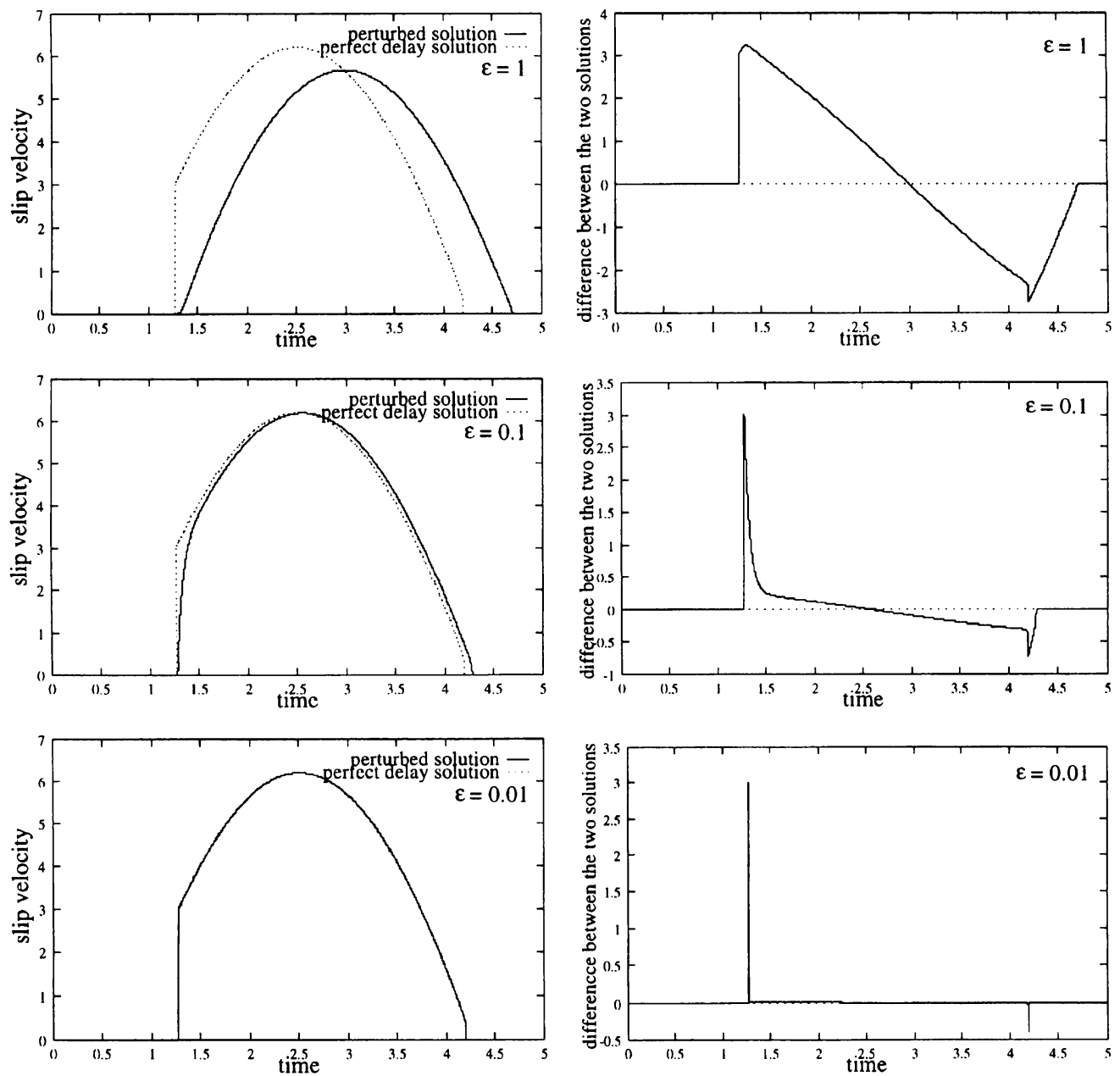

FIG. 3. Perturbed and perfect delay solutions 
interesting to compare the perturbed problem solution and the solution selected with the perfect delay criterion. Figure 3 shows this difference in the example given in the previous section (Eqs. (8), (9)).

One can already see in this figure the close connection between the perfect delay solution and the perturbed one. In the following we prove a convergence result; the right graphs in Fig. 3 point out that the convergence cannot be uniform on $[0, T]$.

5. Basic properties of perturbed solutions. We define the following Cauchy problem associated with the differential inclusion (14):

$$
\left\{\begin{array}{l}
\text { Find } v_{\varepsilon}:[0, T] \longrightarrow \mathbb{R} \text { such that } \\
\left.\left.\varepsilon \frac{d}{d t} v_{\varepsilon}(t) \in \alpha(t)-\beta(v(t)), \text { a.e. on }\right] 0, T\right], \\
v_{\varepsilon}(0)=v^{0},
\end{array}\right.
$$

and we make two elementary assumptions on $\beta$. The first one concerns the regularity of $\beta$ :

\section{Hypothesis 1.}

- $\beta$ is upper semi-continuous with compact convex values,

- $(-\beta)$ is semi-Lipschitz, i.e., there exists a constant $M_{\beta} \in \mathbb{R}$ such that

$$
\left(\beta\left(v_{2}\right)-\beta\left(v_{1}\right)\right)\left(v_{1}-v_{2}\right) \leq M_{\beta}\left(v_{1}-v_{2}\right)^{2}, \quad \forall v_{1}, v_{2} \in \mathbb{R} .
$$

A multi-valued map $\beta$ is upper semi-continuous if for all closed sets $A \subset \mathbb{R}$ the set $\beta^{-1}(A)$ is closed (see [3]). If $\mu: \mathbb{R}^{+} \longrightarrow \mathbb{R}^{+}$is Lipschitz continuous, those conditions are obviously satisfied. The second Hypothesis is that $\beta$ is piecewise strictly monotone and surjective:

Hypothesis 2. There exist $N \geq 0$ and $2 N$ pairs $\left(v_{j}^{*}, \alpha_{j}^{*}\right)_{1 \leq j \leq 2 N}$, with $v_{1}^{*} \leq v_{2}^{*} \leq \ldots \leq$ $v_{2 N}^{*}$ and

- $\left[v_{2 N},+\infty\left[,\left[\alpha_{2 N},+\infty[;]-\infty, v_{1}^{*}\right],\right]-\infty, \alpha_{1}^{*}\right]$ and $\left[v_{2 i}^{*}, v_{2 i+1}^{*}\right],\left[\alpha_{2 i}^{*}, \alpha_{2 i+1}^{*}\right], 1 \leq i \leq$ $N-1$ strictly increasing branches of $\beta$,

- $\left[v_{2 i-1}^{*}, v_{2 i}^{*}\right],\left[\alpha_{2 i-1}^{*}, \alpha_{2 i}^{*}\right], 1 \leq i \leq N$, strictly decreasing branches of $\beta$,

- $\lim _{|v| \rightarrow+\infty}|\beta(v)|=+\infty$,

where $\left[v_{1}, v_{2}\right],\left[\alpha_{1}, \alpha_{2}\right]$ is called a strictly increasing (respectively strictly decreasing) branch of $\beta$ if $\alpha_{1} \in \beta\left(v_{1}\right), \alpha_{2} \in \beta\left(v_{2}\right), \alpha_{1}<\alpha_{2}$ (respectively $\alpha_{1}>\alpha_{2}$ ) and

$$
(\beta(u)-\beta(w))(u-w)>0 \text { (respectively }<0), \forall u, w \in] v_{1}, v_{2}[, u \neq w .
$$

With Hypotheses 1 and 2 , we have $\beta(\mathbb{R})=\mathbb{R}$ and $v \mapsto \beta(v)+M_{\beta} v$ is a maximal monotone map. Often, the dependence of the friction coefficient $\mu$ on $v_{s}$, which is chosen in the models in the literature, is rather simple and sufficiently smooth, and Hypotheses 1 and 2 are not too restrictive.

The existence and uniqueness of an absolutely continuous solution to problem (15), with $\varepsilon>0, \alpha$ measurable and $\beta$ satisfying Hypothesis 1 , is given by a general result on 
semi-Lipschitz differential inclusions, which can be found in [3] for example (moreover, K. Deimling gives in [3] a simple application of differential inclusions to dry friction).

Let us now give a result on the global behavior of the solution of problem (15).

Proposition 1. With $\beta$ satisfying Hypotheses 1 and $2, \alpha \in L^{\infty}(0, T)$ and with a given $\varepsilon>0$, let $\left(v_{1}, \alpha_{1}\right)$ and $\left(v_{2}, \alpha_{2}\right)$ be two pairs of values such that

- $\alpha_{1}<\alpha_{2}, v_{1} \leq v_{2}, \alpha_{1} \in \beta\left(v_{1}\right), \alpha_{2} \in \beta\left(v_{2}\right)$,

- $v^{0} \in\left[v_{1}, v_{2}\right]$,

- $\alpha(t) \in\left[\alpha_{1}, \alpha_{2}\right]$, a.e. on $[0, T]$.

Then the solution $v_{\varepsilon}$ of problem (15) satisfies

$$
v_{\varepsilon}(t) \in\left[v_{1}, v_{2}\right], \quad \forall t \in[0, T] .
$$

Proof. Let $\tilde{\beta}$ be the following multi-valued map:

$$
\tilde{\beta}(v)=\left\{\begin{array}{l}
\left\{\alpha_{1}+\left(v-v_{1}\right)\right\} \text { if } v<v_{1}, \\
\beta(v) \text { if } v \in\left[v_{1}, v_{2}\right] \\
\left\{\alpha_{2}+\left(v-v_{2}\right)\right\} \text { if } v>v_{2} .
\end{array}\right.
$$

Let $\tilde{v_{\varepsilon}}$ be an absolutely continuous solution of the following Cauchy problem:

$$
\left\{\begin{array}{l}
\varepsilon \frac{d}{d t} \tilde{v}_{\varepsilon}(t) \in \alpha(t)-\tilde{\beta}\left(\tilde{v}_{\varepsilon}(t)\right), \text { a.e. on }[0, T], \\
\tilde{v}_{\varepsilon}\left(t_{0}\right)=v^{0} .
\end{array}\right.
$$

Assume that there exists $\left.\left.t_{2} \in\right] 0, T\right]$ such that $\tilde{v}_{\varepsilon}\left(t_{2}\right)<v_{1}$. From the continuity of $\tilde{v}_{\varepsilon}$, there exists $t_{1}<t_{2}$ such that $\tilde{v_{\varepsilon}}\left(t_{1}\right)=v_{1}$ and $\tilde{v_{\varepsilon}}(t)<v_{1}$ for $t_{1}<t \leq t_{2}$. But on this interval:

$$
\tilde{v_{\varepsilon}}(t)=v_{1}+\frac{1}{\varepsilon} \int_{t_{1}}^{t}\left(\alpha(s)-\alpha_{1}+v_{1}-\tilde{v_{\varepsilon}}(s)\right) d s \geq v_{1},
$$

a contradiction. Symmetrically, there does not exist $t_{3} \in[0, T]$ such that $\tilde{v}_{\varepsilon}\left(t_{3}\right)>v_{2}$. Thus $\tilde{v}_{\varepsilon}(t) \in\left[v_{1}, v_{2}\right]$ for all $t \in[0, T]$, and $\tilde{v}_{\varepsilon}$ is a solution of problem (15) because $\tilde{\beta}$ and $\beta$ are identical on $\left[v_{1}, v_{2}\right]$. The uniqueness of the solution of problem (15) implies $\tilde{v_{\varepsilon}}=v_{\varepsilon}$.

Proposition 2. With $\beta$ satisfying Hypotheses 1 and $2, \alpha \in L^{\infty}(0, T)$ and with a given $\varepsilon>0$, the solution $v_{\varepsilon}$ of problem (15) has the following properties:

(1) $\left\|v_{\varepsilon}\right\|_{L^{\infty}(0, T)}<M_{1}$, where $M_{1}>0$ is a constant independent of $\varepsilon$,

(2) $\frac{d}{d t} v_{\varepsilon} \in L^{\infty}(0, T)$.

Proof. Let us set

$$
\begin{aligned}
& \alpha_{1}=\min \left(\alpha_{1}^{*}, \underset{t \in[0, T]}{\operatorname{essinf}} \alpha(t), \beta\left(v^{0}\right)\right), \quad \alpha_{2}=\max \left(\alpha_{2 N}^{*}, \underset{t \in[0 . T]}{\operatorname{ess} \sup } \alpha(t), \beta\left(v^{0}\right)\right), \\
& v_{1}=\min \left(\beta^{-1}\left(\alpha_{1}\right)\right), \quad v_{2}=\max \left(\beta^{-1}\left(\alpha_{2}\right)\right) .
\end{aligned}
$$

Then $v_{1} \leq v_{2}$ and Proposition 1 implies

$$
v_{\varepsilon}(t) \in\left[v_{1}, v_{2}\right], \quad \forall t \in[0, T] .
$$

Properties 1 and 2 are immediate consequences of this result. 
6. Boundary layer at $\mathbf{t}=\mathbf{0}$. The first step in establishing the convergence result is to analyze the behavior of the sequence $v_{\varepsilon}$ on a small time interval near the initial value. The goal of this section is to prove that the sequence $v_{\varepsilon}$ converges pointwise in a neighborhood of $t=0$. In order to do this, we introduce some concepts inspired by the work of Tikhonov et al. [13]. The pointwise convergence at $t=0$ is obvious because $v_{\varepsilon}(0)=v^{0}$ for all $\varepsilon>0$; in spite of this, if the initial condition satisfies

$$
\alpha^{+}(0) \notin \beta\left(v^{0}\right),
$$

there is a boundary layer behavior at $t=0$ of the sequence of solution $v_{\varepsilon}$ when $\varepsilon$ tends to zero. Let us introduce the terminology concerning the points of the graph $\beta$ when $\beta$ satisfies Hypothesis 2:

Terminology. A point $(\alpha, v)$ with $\alpha \in \beta(v)$ is called a stable point (respectively an unstable point) if there exists a strictly increasing branch (respectively strictly decreasing branch) $\left[v_{j}^{*}, v_{j+1}^{*}\right],\left[\alpha_{j}^{*}, \alpha_{j+1}^{*}\right]$ with $\left.\alpha \in\right] \alpha_{j}^{*}, \alpha_{j+1}^{*}\left[(\right.$ respectively $\alpha \in] \alpha_{j+1}^{*}, \alpha_{j}^{*}[)$ and $v \in$ $\left[v_{j}^{*}, v_{j+1}^{*}\right]$. Points $\left(\alpha_{j}^{*}, v_{j}^{*}\right)$ are called critical points; points $\left(\alpha_{2 i-1}^{*}, v_{2 i-1}^{*}\right)$ are the critical maximum points; points $\left(\alpha_{2 i}^{*}, v_{2 i}^{*}\right)$ are the critical minimum points.

In the following, the data $\alpha$ is taken with bounded variation $(\alpha \in B V(0, T)$ ). For basic properties of functions with bounded variations we refer to [4] or [11]. For basic properties of multi-valued maps we refer to [3].

6.1. Cluster point of the boundary layer.

Definition 1. A point $v \in \mathbb{R}$ is called a cluster point of the boundary layer at $t=0$ for problem (15) if and only if there exist a strictly positive sequence $\left(\varepsilon_{n}\right)_{n \geq 0}$ and a positive sequence $\left(t_{n}\right)_{n \geq 0}$ such that

$$
\lim _{n \rightarrow+\infty} \varepsilon_{n}=0, \quad \lim _{n \rightarrow+\infty} t_{n}=0, \quad \lim _{n \rightarrow+\infty} v_{\varepsilon_{n}}\left(t_{n}\right)=v
$$

This means that the pair $(0, v)$ is a cluster point of the graph sequence $\left(t, v_{\varepsilon}(t)\right)$.

Proposition 3. With $\alpha \in B V(0, T)$ and $\beta$ satisfying Hypotheses 1 and 2, the set of all cluster points of the boundary layer at $t=0$ for problem (15) is a closed bounded interval. We denote this interval by $\mathrm{I}_{a}\left(v^{0}\right)$.

Proof. From Proposition 2 we know that the sequence $v_{\varepsilon}$ is bounded in $L^{\infty}(0, T)$, uniformly in $\varepsilon$. Thus the set of all cluster points is necessarily bounded. It is closed because it is the intersection of the closed set $t=0$ with the closed set of all cluster points of the graph sequence $\left(t, v_{\varepsilon}(t)\right)$. The value $v^{0}$ is always a cluster point. If there exists another cluster point $v>v^{0}$ then, from the definition of a cluster point, there exist a strictly positive sequence $\left(\varepsilon_{n}\right)_{n \geq 0}$ and a positive sequence $\left(t_{n}\right)_{n \geq 0}$ with

$$
\lim _{n \rightarrow+\infty} \varepsilon_{n}=0, \quad \lim _{n \rightarrow+\infty} t_{n}=0, \quad \lim _{n \rightarrow+\infty} v_{\varepsilon_{n}}\left(t_{n}\right)=v
$$

If we set

$$
\zeta_{n}=v_{\varepsilon_{n}}\left(t_{n}\right)-v,
$$

then $\lim _{n \rightarrow+\infty} \zeta_{n}=0$; and for any $\left.\tilde{v} \in\right] v^{0}, v\left[\right.$, there exists $\tilde{N}>0$ such that $\left|\zeta_{n}\right|<v-\tilde{v}$ for $n>\tilde{N}$. From the continuity of the functions $v_{\varepsilon_{n}}$, there exists $\tilde{t}_{\varepsilon_{n}} \in\left[0, t_{n}\right]$, for $n>\tilde{N}$, 
such that

$$
v_{\varepsilon_{n}}\left(\tilde{t}_{\varepsilon_{n}}\right)=\tilde{v}
$$

Thus $\lim _{n \rightarrow+\infty} v_{\varepsilon_{n}}\left(\tilde{t}_{\varepsilon_{n}}\right)=\tilde{v}$, and $\tilde{v}$ is a cluster point for the same sequences as $v$. The same proof holds when there exists a cluster point $v<v^{0}$, and this implies that the set of all the cluster points is an interval.

We present now two lemmas which will be very useful in the following.

Lemma 1. Let $\beta$ satisfy Hypotheses 1 and 2 , and $\alpha \in B V(0, T)$. If there exist $v_{0} \in \mathbb{R}$ and $t_{0} \in[0, T[$ such that

$$
\alpha^{+}\left(t_{0}\right)>\beta\left(v_{0}\right)
$$

then there exist $\delta>0$ and $\zeta>0$ such that

$$
\alpha^{+}(t)>\beta(v), \quad \forall t \in\left[t_{0}, t_{0}+\zeta\right], \forall v \in\left[v_{0}-\delta, v_{0}+\delta\right] .
$$

Proof. Let us denote by

$$
\eta=\min \left(\alpha^{+}\left(t_{0}\right)-\beta\left(v_{0}\right)\right)
$$

the gap between $\alpha^{+}\left(t_{0}\right)$ and $\beta\left(v_{0}\right)$. Since the right limit $\alpha^{+}\left(t_{0}\right)$ exists, there exists $\zeta>0$ such that

$$
\left|\alpha^{+}(t)-\alpha^{+}\left(t_{0}\right)\right|<\frac{\eta}{4}, \quad \forall t \in\left[t_{0}, t_{0}+\zeta\right]
$$

Assume that

$$
\forall \delta>0, \exists v_{\delta} \in\left[v_{0}-\delta, v_{0}+\delta\right] ; \min \left(\alpha^{+}\left(t_{0}\right)-\beta\left(v_{\delta}\right)\right)<\frac{\eta}{4} .
$$

Then we have

$$
\left.\left.\lim _{\delta \rightarrow 0} v_{\delta}=v_{0}, \text { and }\left\{\alpha^{+}\left(t_{0}\right)-\beta\left(v_{\delta}\right)\right\} \cap\right]-\infty, \frac{\eta}{4}\right] \neq \emptyset .
$$

Thus, the upper semi-continuity of $\beta$ implies

$$
\left.\left.\left\{\alpha^{+}\left(t_{0}\right)-\beta\left(v_{0}\right)\right\} \cap\right]-\infty, \frac{\eta}{4}\right] \neq \emptyset,
$$

a contradiction. So there exists $\delta>0$ such that

$$
\alpha^{+}\left(t_{0}\right)>\beta(v)+\frac{3 \eta}{4}, \quad \forall v \in\left[v_{0}-\delta, v_{0}+\delta\right] .
$$

The second lemma gives information on the sign of $\beta(v)-\alpha^{+}(0)$ on the interval $\mathrm{I}_{a}\left(v^{0}\right)$ :

Lemma 2. If $\beta$ satisfies Hypotheses 1 and 2 , and $\alpha \in B V(0, T)$, then

$$
\min \left[\left(\beta(v)-\alpha^{+}(0)\right)\left(v-v^{0}\right)\right] \leq 0, \quad \forall v \in \mathrm{I}_{a}\left(v^{0}\right) .
$$

Proof. Assume that there exists $v_{b} \in \mathrm{I}_{a}\left(v^{0}\right)$ such that $\left(\beta\left(v_{b}\right)-\alpha^{+}(0)\right)\left(v_{b}-v^{0}\right)>0$. Suppose for simplicity that $v_{b}>v^{0}$ (the case $v_{b}<v^{0}$ is similar). Then we have

$$
\beta\left(v_{b}\right)-\alpha^{+}(0)>0 .
$$

From Lemma 1 , there exist $\delta>0$ and $0<\zeta<v_{b}-v^{0}$ such that

$$
\beta(v)-\alpha^{+}(t)>0, \quad \forall t \in[0, \zeta], \forall v \in\left[v_{b}-\delta, v_{b}+\delta\right] .
$$


Thus, for any $\varepsilon>0$ we have

$$
\left.\left.\frac{d}{d t} v_{\varepsilon}(t)<0 \text {, a.e. on }\right] 0, \zeta\right] \text { and whenever } v_{\varepsilon}(t) \in\left[v_{b}-\delta, v_{b}+\delta\right] \text {. }
$$

We can conclude that $v_{\varepsilon}(t) \leq v_{b}-\delta$ for all $t \in[0, \zeta]$, because $v_{\varepsilon}(0)<v_{b}-\delta$. So the point $v_{b}$ cannot be a cluster point, a contradiction.

6.2. Limit points of the boundary layer.

Definition 2. A point $v \in \mathbb{R}$ is called a limit point of the boundary layer at $t=0$ for the problem (15) if and only if there exists $t_{\varepsilon}>0$ for all $\varepsilon>0$ such that

$$
\lim _{\varepsilon \rightarrow 0} t_{\varepsilon}=0, \quad \lim _{\varepsilon \rightarrow 0} v_{\varepsilon}\left(t_{\varepsilon}\right)=v .
$$

Proposition 4. With $\alpha \in B V(0, T)$ and $\beta$ satisfying Hypotheses 1 and 2 , the set of all limit points of the boundary layer at $t=0$ for problem (15) is a closed sub-interval of $\mathrm{I}_{a}\left(v^{0}\right)$. We denote this interval by $\mathrm{I}_{l}\left(v^{0}\right)$.

The proof of this proposition is similar to that of Proposition 3.

Proposition 5. With $\alpha \in B V(0, T)$ and $\beta$ satisfying Hypotheses 1 and 2 , the initial value $v^{0}$ is always an extremity of the interval $\mathrm{I}_{l}\left(v^{0}\right)$. If $\left(\alpha^{+}(0), v^{0}\right)$ is not an unstable point of $\beta$, then $v^{0}$ is also an extremity of the interval $\mathrm{I}_{a}\left(v^{0}\right)$.

Proof. Let us set $\mathrm{I}_{a}\left(v^{0}\right)=\left[v_{a}, v_{b}\right]$ and assume $\left.v^{0} \in\right] v_{a}, v_{b}[$. From Lemma 2 we have

$$
\begin{array}{ll}
\alpha^{+}(0) \leq \max \beta(v), & \forall v \in\left[v_{a}, v^{0}[,\right. \\
\alpha^{+}(0) \geq \min \beta(v), & \forall v \in] v^{0}, v_{b}[.
\end{array}
$$

It means that $\left(\alpha^{+}(0), v^{0}\right)$ is an unstable point of $\beta$. Let $\left[v_{i}^{*}, v_{i+1}^{*}\right],\left[\alpha_{i}^{*}, \alpha_{i+1}^{*}\right]$ be the corresponding decreasing branch of $\beta$ and set

$$
\left[v_{a}^{\prime}, v_{b}^{\prime}\right]=\left[v_{i}^{*}, v_{i+1}^{*}\right] \cap \mathbf{I}_{l}\left(v^{0}\right) .
$$

Then $\left.v^{0} \in\right] v_{a}^{\prime}, v_{b}^{\prime}[$, and we have

$$
\alpha^{+}(0)<\beta\left(v_{a}^{\prime}\right), \quad \alpha^{+}(0)>\beta\left(v_{b}^{\prime}\right) .
$$

From Lemma 1 , there exist $\delta>0$ and $\zeta>0$ such that

$$
\begin{array}{ll}
\alpha^{+}(t)<\beta(v), & \text { for } v \in\left[v_{a}^{\prime}-\delta, v_{a}^{\prime}+\delta\right], \text { and } t \in[0, \zeta], \\
\alpha^{+}(t)>\beta(v), & \text { for } v \in\left[v_{b}^{\prime}-\delta, v_{b}^{\prime}+\delta\right], \text { and } t \in[0, \zeta] .
\end{array}
$$

Because $v_{a}^{\prime}$ and $v_{b}^{\prime}$ are limit points, there exists $\varepsilon_{0}>0$ such that for all $\left.\varepsilon \in\right] 0, \varepsilon_{0}$ ] we have

$$
\left.\left.\exists t_{\varepsilon}^{a}, t_{\varepsilon}^{b} \in\right] 0, t_{0}\right], \quad\left|v_{\varepsilon}\left(t_{\varepsilon}^{a}\right)-v_{a}^{\prime}\right|<\frac{\delta}{2}, \quad\left|v_{\varepsilon}\left(t_{\varepsilon}^{b}\right)-v_{b}^{\prime}\right|<\frac{\delta}{2} .
$$

For a given $\left.\varepsilon \in] 0, \varepsilon_{0}\right]$, assume $t_{\varepsilon}^{a}>t_{\varepsilon}^{b}$. We have $\alpha(t)-\beta(v)>0$ for $\left.\left.t \in\right] 0, \zeta\right]$ and for $v \in\left[v_{b}^{\prime}-\delta, v_{b}^{\prime}+\delta\right]$; thus also $\frac{d}{d t} v_{\varepsilon}>0$ a.e. on $[0, \zeta]$ and whenever $v_{\varepsilon} \in\left[v_{b}^{\prime}-\delta, v_{b}^{\prime}+\delta\right]$. So $\left|v_{\varepsilon}\left(t_{\varepsilon}^{a}\right)-v_{a}^{\prime}\right|<\frac{\delta}{2}$ is not possible. In the same way, if $t_{\varepsilon}^{a}<t_{\varepsilon}^{b}$, then it is not possible to have $\left|v_{\varepsilon}\left(t_{\varepsilon}^{b}\right)-v_{b}^{\prime}\right|<\frac{\delta}{2}$, a contradiction. 


\subsection{Attractive point of the boundary layer.}

Definition 3. A point $v_{0} \in \mathbb{R}$ is called an attractive point at $t_{0} \in[0, T$ [ for problem (15) if the following two conditions hold:

- $\alpha^{+}\left(t_{0}\right) \in \beta\left(v_{0}\right)$,

- there exist a strictly increasing branch $\left[v_{1}, v_{2}\right],\left[\alpha_{1}, \alpha_{2}\right]$ of $\beta$ and $\zeta>0$ such that

$$
v_{0} \in\left[v_{1}, v_{2}\right], \quad \alpha^{+}(t) \in\left[\alpha_{1}, \alpha_{2}\right], \quad \forall t \in\left[t_{0}, t_{0}+\zeta[.\right.
$$

If $\left(\alpha^{+}(0), v_{0}\right)$ is a stable point of $\beta$ then $v_{0}$ is an attractive point. The limitation of the second condition operates when $\left(\alpha^{+}(0), v_{0}\right)$ is a critical point.

Definition 4. A point $v_{0} \in \mathbb{R}$ is called a backward attractive point at $\left.\left.t_{0} \in\right] 0, T\right]$ for the problem (15) if the following two conditions hold:

- $\alpha^{-}\left(t_{0}\right) \in \beta\left(v_{0}\right)$,

- there exist a strictly increasing branch $\left[v_{1}, v_{2}\right],\left[\alpha_{1}, \alpha_{2}\right]$ of $\beta$ and $\zeta>0$ such that

$$
\left.\left.v_{0} \in\left[v_{1}, v_{2}\right], \quad \alpha^{-}(t) \in\left[\alpha_{1}, \alpha_{2}\right], \quad \forall t \in\right] t_{0}-\zeta, t_{0}\right] .
$$

It is possible to characterize the behavior of the sequence of solutions $v_{\varepsilon}$ when the interval $\mathrm{I}_{a}\left(v^{0}\right)$ contains an attractive point as follows:

Lemma 3. With $\alpha \in B V(0, T)$ and $\beta$ satisfying Hypotheses 1 and 2 , if the interval $\mathrm{I}_{a}\left(v^{0}\right)$ contains an attractive point $v_{0}$, then for all strictly positive sequences $\left(\varepsilon_{n}\right)_{n \geq 0}$ and all positive sequences $\left(t_{n}\right)_{n \geq 0}$ such that

$$
\lim _{n \rightarrow+\infty} \varepsilon_{n}=0, \quad \lim _{n \rightarrow+\infty} t_{n}=0, \quad \lim _{n \rightarrow+\infty} v_{\varepsilon_{n}}\left(t_{n}\right)=v_{0}
$$

for all $\delta>0$, there exists $\zeta_{2}>0$ such that for all $\left.\zeta_{1} \in\right] 0, \zeta_{2}[$ there exists $\tilde{N}>0$ with

$$
\begin{gathered}
v_{\varepsilon_{n}}(t) \in\left[v_{0}-\delta, v_{0}+\delta\right], \forall t \in\left[\zeta_{1}, \zeta_{2}\right], \forall n \geq \tilde{N}, \\
\left(v_{\varepsilon_{n}}(t)-v_{0}\right) \operatorname{Sgn}\left(v_{0}-v^{0}\right)<\delta, \forall t \in\left[0, \zeta_{2}\right], \forall n \geq \tilde{N} .
\end{gathered}
$$

Proof. Let us assume $v_{0}>v^{0}$ (if $v_{0}=v^{0}$ the result is an obvious application of Proposition 1 and if $v_{0}<v^{0}$ the proof is similar). Because $v_{0}$ is an attractive point, there exists a strictly increasing branch $\left[v_{i}^{*}, v_{i+1}^{*}\right],\left[\alpha_{i}^{*}, \alpha_{i+1}^{*}\right]$ of $\beta$ and $\zeta>0$ such that

$$
v_{0} \in\left[v_{1}, v_{2}\right], \quad \alpha^{+}(t) \in\left[\alpha_{1}, \alpha_{2}\right], \quad \forall t \in[0, \zeta] .
$$

If $v_{0}=v_{i}^{*}$ and $\alpha^{+}(0)=\alpha_{1}$, then $\left(\alpha^{+}(0), v_{0}\right)$ is a critical minimum attractive point. Because $\left[v_{i-1}^{*}, v_{i}^{*}\right],\left[\alpha_{i-1}^{*}, \alpha_{i}^{*}\right]$ is a strictly decreasing branch of $\beta$, there exists $\left.v \in\right] v^{0}, v_{0}$ [ such that $\beta(v)>\alpha^{+}(0)$, a contradiction from Lemma 2. Thus $\left(\alpha^{+}(0), v_{0}\right)$ is not a critical minimum point and there exists $\delta_{0}>0$ such that

$$
\alpha^{+}(0)>\beta(v), \quad \forall v \in\left[v_{0}-\delta, v_{0}[.\right.
$$

Given any $\delta>0$, let us set

$$
\begin{aligned}
\tilde{v}_{1}=v_{0}-\min \left(\delta_{0}, \delta\right), & \tilde{\alpha}_{1} \in \beta\left(\tilde{v_{1}}\right), \\
\tilde{v}_{2}=v_{0}+\min \left(v_{2}-v_{0}, \delta\right), & \tilde{\alpha}_{2}=\max \beta\left(\tilde{v}_{2}\right) .
\end{aligned}
$$

Because of the existence of the right limit $\alpha^{+}(0)$, there exists $\zeta_{2}>0$ such that

$$
\alpha^{+}(t) \in\left[\tilde{\alpha}_{1}, \tilde{\alpha}_{2}\right], \quad \forall t \in\left[0, \zeta_{2}\right] .
$$


Moreover, if we consider $\left(\varepsilon_{n}\right)_{n \geq 0}$ strictly positive and $\left(t_{n}\right)_{n \geq 0}$ positive verifying

$$
\lim _{n \rightarrow+\infty} \varepsilon_{n}=0, \quad \lim _{n \rightarrow+\infty} t_{n}=0, \quad \lim _{n \rightarrow+\infty} v_{\varepsilon_{n}}\left(t_{n}\right)=v
$$

then for all $\left.\left.\zeta_{1} \in\right] 0, \zeta_{2}\right]$, there exists $\tilde{N}>0$ such that

$$
t_{n}<\zeta_{2}, \quad \forall n \geq \tilde{N}, \quad v_{\varepsilon}\left(t_{n}\right) \geq \tilde{v}_{1} .
$$

Let $\left.\left.\tilde{t}_{n} \in\right] 0, t_{n}\right]$ be the smallest time $t$ such that $v_{\varepsilon}(t)=v_{0}-\delta$. We can apply Proposition 1 on intervals $\left[\tilde{v}_{1}, \tilde{v}_{2}\right]$ and $\left[\tilde{\alpha}_{1}, \tilde{\alpha}_{2}\right]$ in order to conclude

$$
\begin{aligned}
& v_{\varepsilon}(t) \in\left[\tilde{v}_{1}, \tilde{v}_{2}\right], \quad \forall t \in\left[\zeta_{1}, \zeta_{2}\right], \\
& v_{\varepsilon}(t) \leq \tilde{v}_{2}, \quad \forall t \in\left[0, \zeta_{2}\right] . \quad \square
\end{aligned}
$$

LEMmA 4. With $\alpha \in B V(0, T)$ and $\beta$ satisfying Hypotheses 1 and 2 , if the interval $\mathrm{I}_{l}\left(v^{0}\right)$ contains an attractive point $v_{0}$, then for all $\delta>0$ there exists $\zeta_{2}>0$ such that for all $\left.\zeta_{1} \in\right] 0, \zeta_{2}\left[\right.$ there exists $\varepsilon_{0}>0$ with

$$
\begin{gathered}
\left.\left.v_{\varepsilon}(t) \in\left[v_{0}-\delta, v_{0}+\delta\right], \forall t \in\left[\zeta_{1}, \zeta_{2}\right], \forall \varepsilon \in\right] 0, \varepsilon_{0}\right], \\
\left.\left.\left(v_{\varepsilon}(t)-v_{0}\right) \operatorname{Sgn}\left(v_{0}-v^{0}\right)<\delta, \forall t \in\left[0, \zeta_{2}\right], \forall \varepsilon \in\right] 0, \varepsilon_{0}\right] .
\end{gathered}
$$

The proof of this lemma is similar to the proof of the previous lemma.

Proposition 6. With $\alpha \in B V(0, T)$ and $\beta$ satisfying Hypotheses 1 and 2 , if we set $\mathrm{I}_{a}\left(v^{0}\right)=\left[i_{a 1}, i_{a 2}\right]$ and $\mathrm{I}_{l}\left(v^{0}\right)=\left[i_{l 1}, i_{l 2}\right]$, then we have the following properties:

(a) the interval $] i_{a 1}, i_{a 2}$ [ does not contain any attractive points,

(b) $\alpha^{+}(0) \geq \max \left(\beta\left(i_{a 1}\right), \beta\left(i_{l 1}\right)\right)$ and $\alpha^{+}(0) \leq \min \left(\beta\left(i_{a 2}\right), \beta\left(i_{l 2}\right)\right)$,

(c) if $\left(\alpha^{+}(0), i_{a 1}\right)$ or $\left(\alpha^{+}(0), i_{l 1}\right)$ is a critical minimum point, then it is attractive; if $\left(\alpha^{+}(0), i_{a 2}\right)$ or $\left(\alpha^{+}(0), i_{l 2}\right)$ is a critical maximum point, then it is attractive,

(d) each extremity of $\mathrm{I}_{a}\left(v^{0}\right)$ or $\mathrm{I}_{l}\left(v^{0}\right)$ is either $v^{0}$ or an attractive point.

Proof. (a) Assume there exists an attractive point $v_{0} \in\left[v^{0}, i_{a 2}[\right.$. Applying Lemma 3 with $\delta=\frac{i_{a 2}-v_{0}}{2}$ we obtain that $i_{a 2}$ is not a cluster point, a contradiction. In the same way, the interval $\left.] i_{a 1}, v^{0}\right]$ does not contain any attractive points.

(b) Assume $\alpha^{+}(0)>\beta\left(i_{a 2}\right)$ (the other cases are analogous). From Lemma 1, there exist $\delta>0$ and $\zeta>0$ such that

$$
\alpha^{+}(t)>\beta(v), \quad \forall v \in\left[i_{a 2}-\delta, i_{a 2}+\delta\right], \quad \forall t \in[0, \zeta] .
$$

Let us set

$$
\eta=\min _{\substack{v \in\left[i_{a 2}-\delta, i_{a 2}+\delta\right] \\ t \in[0, \zeta]}}\left(\alpha^{+}(t)-\beta(v)\right) .
$$

Because $i_{a 2}$ is a cluster point, there exist a strictly positive sequence $\left(\varepsilon_{n}\right)_{n \geq 0}$ and a positive sequence $\left(t_{n}\right)_{n \geq 0}$ such that

$$
\lim _{n \rightarrow+\infty} \varepsilon_{n}=0, \quad \lim _{n \rightarrow+\infty} t_{n}=0, \quad \lim _{n \rightarrow+\infty} v_{\varepsilon_{n}}\left(t_{n}\right)=i_{a 2} .
$$


For all $\theta \in] 0, \zeta]$ there exists $\tilde{N} \geq 0$ such that for all $n \geq \tilde{N}$ we have

But

$$
\left.\left.v_{\varepsilon_{n}}\left(t_{n}\right) \in\left[i_{a 2}-\delta, i_{a 2}+\delta\right], \quad t_{n} \in\left[0, \frac{\theta}{2}\right], \quad \varepsilon_{n} \in\right] 0, \frac{\eta \theta}{\delta}\right] .
$$

$$
\varepsilon \frac{d}{d t} v_{\varepsilon_{n}}(t) \in \alpha(t)-\beta\left(v_{\varepsilon_{n}}(t) \geq \eta \text {, a.e. on }[0, \zeta] \text { and whenever } v_{\varepsilon_{n}}(t) \in\left[i_{a 2}-\delta, i_{a 2}+\delta\right]\right. \text {. }
$$

Thus, for a given $n \geq \tilde{N}$ we have

$$
v_{\varepsilon_{n}}(\theta) \geq \min \left(i_{a 2}-\delta+\frac{\eta}{\varepsilon_{n}}\left(\theta-t_{n}\right), i_{a 2}+\delta\right) .
$$

In particular,

$$
v_{\varepsilon_{n}}(\theta) \geq i_{a 2}+\delta .
$$

From the continuity of $v_{\varepsilon_{n}}$, there exists $\tilde{t}_{n} \in[0, \theta]$ such that $v_{\varepsilon_{n}}\left(\tilde{t}_{n}\right)=i_{a 2}+\delta$. Thus $i_{a 2}+\delta$ is a cluster point, a contradiction.

(c) Assume $\left(\alpha^{+}(0), i_{a 2}\right)$ is a critical maximum but non-attractive point (the other cases are analogous). There exists $\delta>0$ such that $\left[i_{a 2}-\delta, i_{a 2}\right]$ is a strictly increasing branch of $\beta$ and $\left[i_{a 2}, i_{a 2}+\delta\right]$ is a strictly decreasing branch of $\beta$. Existence of the right limit $\alpha^{+}(0)$ implies that there exists $\zeta>0$ such that

$$
\alpha^{+}(t)>\beta\left(i_{a 2}-\delta\right), \quad \forall t \in[0, \zeta] .
$$

The point $\left(\alpha^{+}(0), i_{a 2}\right)$ is not an attractive point since

$$
\forall \theta \in] 0, \zeta], \exists 0<t_{\theta}<\tilde{t}_{\theta}<\theta, \quad \alpha^{+}(t)>\beta(v), \quad \forall v \in\left[i_{a 2}-\delta, i_{a 2}+\delta\right], \quad \forall t \in\left[t_{\theta}, \tilde{t}_{\theta}\right] .
$$

The point $i_{a 2}$ is a cluster point. Thus there exist a strictly positive sequence $\left(\varepsilon_{n}\right)_{n \geq 0}$ and a positive sequence $\left(t_{n}\right)_{n \geq 0}$ such that

$$
\lim _{n \rightarrow+\infty} \varepsilon_{n}=0, \quad \lim _{n \rightarrow+\infty} t_{n}=0, \quad \lim _{n \rightarrow+\infty} v_{\varepsilon_{n}}\left(t_{n}\right)=i_{a 2} .
$$

For a given $\theta \in] 0, \zeta]$ let us set

$$
\eta=\min \left(\alpha^{+}(t)-\beta(v)\right), \quad \text { for } v \in\left[i_{a 2}-\delta, i_{a 2}+\delta\right], \quad \text { and } t \in\left[t_{\theta}, \tilde{t}_{\theta}\right] \text {. }
$$

There exists $\tilde{N} \geq 0$ such that for all $n \geq \tilde{N}$ we have

$$
\left.\left.v_{\varepsilon_{n}}\left(t_{n}\right) \in\left[i_{a 2}-\delta, i_{a 2}+\delta\right], \quad t_{n} \in\left[0, t_{\theta}\right], \quad \varepsilon_{n} \in\right] 0, \frac{\eta\left(\tilde{t}_{\theta}-t_{\theta}\right)}{2 \delta}\right] .
$$

In particular,

$$
v_{\varepsilon_{n}}(t) \geq i_{a 2}-\delta, \quad \forall t \in\left[t_{n}, t_{\theta}\right]
$$

Then

$$
\frac{d}{d t} v_{\varepsilon_{n}}(t) \geq \frac{\eta}{\varepsilon_{n}}, \quad \text { a.e. on }\left[t_{\theta}, \tilde{t}_{\theta}\right], \text { and whenever } v_{\varepsilon_{n}} \leq i_{a 2}+\delta
$$

We obtain

$$
v_{\varepsilon_{n}}\left(\tilde{t}_{\theta}\right) \geq \min \left(i_{a 2}+\delta, i_{a 2}-\delta \frac{\eta}{\varepsilon_{n}}\left(\tilde{t}_{\theta}-t_{\theta}\right)\right)
$$

So

$$
v_{\varepsilon_{n}}\left(\tilde{t}_{\theta}\right) \geq i_{a 2}+\delta \text {. }
$$


From the continuity of $v_{\varepsilon_{n}}$, there exists a $\tilde{t}_{n} \in\left[0, \tilde{t}_{\theta}\right]$ such that $v_{\varepsilon_{n}}\left(\tilde{t}_{n}\right)=i_{a 2}+\delta$. Thus $i_{a 2}+\delta$ is a cluster point, a contradiction. (d) Assume for instance that $i_{a 2}$ is neither equal to $v^{0}$ nor an attractive point. A consequence of property $\mathrm{b}$ ) of Lemma 2 is that $\alpha^{+}(0) \in \beta\left(i_{a 2}\right)$. The point $\left(i_{a 2}, \alpha^{+}(0)\right)$ cannot be an unstable point and if it is a critical point, property $c$ ) implies that it is necessarily an attractive critical maximum point. If it is not a critical point, it is a stable point which is automatically attractive.

The previous proposition allows us to describe the intervals $\mathrm{I}_{a}\left(v^{0}\right)$ and $\mathrm{I}_{l}\left(v^{0}\right)$ whenever $\left(\alpha^{+}(0), v^{0}\right)$ is not an unstable point of $\beta$, as follows:

- if $\left(\alpha^{+}(0), v^{0}\right)$ is an attractive point, then $\mathrm{I}_{a}\left(v^{0}\right)=\mathrm{I}_{l}\left(v^{0}\right)=\left\{v^{0}\right\}$;

- if either $\alpha^{+}(0)>\beta\left(v^{0}\right)$ or $\left(\alpha^{+}(0), v^{0}\right)$ is a critical maximum non-attractive point, then

$$
\mathrm{I}_{a}\left(v^{0}\right)=\mathrm{I}_{l}\left(v^{0}\right)=\left[v^{0}, \min \left\{v>v^{0}: v \text { attractive point }\right\}\right]
$$

- if either $\alpha^{+}(0)<\beta\left(v^{0}\right)$ or $\left(\alpha^{+}(0), v^{0}\right)$ is a critical minimum non-attractive point, then

$$
\mathrm{I}_{a}\left(v^{0}\right)=\mathrm{I}_{l}\left(v^{0}\right)=\left[\max \left\{v<v^{0}: v \text { attractive point }\right\}, v^{0}\right] .
$$

In particular, when $\left(\alpha^{+}(0), v^{0}\right)$ is not an unstable point of $\beta, \mathrm{I}_{l}\left(v^{0}\right)=\mathrm{I}_{a}\left(v^{0}\right)$ and this interval contains a unique attractive point.

Let us briefly consider the case where $\left(\alpha^{+}(0), v^{0}\right)$ is an unstable point of $\beta$. This corresponds to an initial value which is on the border between two regions of attraction. The behavior of the solution depends on the behavior of $\alpha(t)$ in a neighborhood of $t=0$. If there exists a small interval $] 0, \zeta]$ on which

- $\alpha$ is constant, then the unique solution on this interval is $v_{\varepsilon} \equiv v^{0}$ for all $\varepsilon>0$;

- $\alpha$ is strictly increasing, then $\mathrm{I}_{a}\left(v^{0}\right)=\mathrm{I}_{l}\left(v^{0}\right)=\left[v^{0}, \min \left\{v>v^{0}: v\right.\right.$ attractive point $\left.\}\right]$,

- $\alpha$ is decreasing, then $\mathrm{I}_{a}\left(v^{0}\right)=\mathrm{I}_{l}\left(v^{0}\right)=\left[\max \left\{v<v^{0}: v\right.\right.$ attractive point $\left.\}, v^{0}\right]$.

On the other hand, if $\alpha$ does not verify any of these three conditions, for instance this is the case with

$$
\alpha(t)=\alpha(0)+t^{3} \sin \left(\frac{1}{t}\right),
$$

then it is possible to have $\mathrm{I}_{a}\left(v^{0}\right)=\left[\max \left\{v<v^{0}: v\right.\right.$ attractive point $\}, \min \left\{v>v^{0}\right.$ : $v$ attractive point $\}]$ and $\mathrm{I}_{l}\left(v^{0}\right)=\left\{v^{0}\right\}$. So $\mathrm{I}_{a}\left(v^{0}\right)$ contains two attractive points and $\mathrm{I}_{l}\left(v^{0}\right)$ none. In this case, the sequence $v_{\varepsilon}$ does not converge. To avoid this, we impose in the following the condition that $\left(\alpha^{+}(0), v^{0}\right)$ is not an unstable point, even though this condition is somewhat too strong.

6.4. Region of attraction of an attractive point.

DEFINITION 5. Let $v_{0}$ be an attractive point of the boundary layer at $t=0$ for problem (15). We call the region of attraction of $v_{0}$ the interval of all the initial values $v^{0}$ such that $v_{0} \in \mathrm{I}_{l}\left(v^{0}\right)$. We denote this interval by $\mathrm{I}_{b}\left(v_{0}\right)$.

It is easy to obtain the expression for the region of attraction from the expression for $\mathrm{I}_{l}\left(v^{0}\right)$. The interior of $\mathrm{I}_{b}\left(v_{0}\right)$ is $] v_{a}, v_{b}[$ with

$$
v_{a}=\mid \begin{array}{ll}
v_{0} \text { if }\left(\alpha^{+}(0), v_{0}\right) \text { is a critical minimum, } \\
\max \left\{\left\{v<v_{0}:\left(\alpha^{+}(0), v\right) \text { unstable or critical maximum attractive }\right\}\right. & \cup\{-\infty\}\} \\
& \text { otherwise, }
\end{array}
$$


$v_{b}=\mid \begin{array}{ll}v_{0} \text { if }\left(\alpha^{+}(0), v_{0}\right) \text { is a critical maximum, } \\ \min \left\{\left\{v>v_{0}:\left(\alpha^{+}(0), v\right) \text { unstable or critical minimum attractive }\right\}\right. & \cup\{+\infty\}\} \\ & \text { otherwise. }\end{array}$

Clearly, with $v^{0} \in \mathrm{I}_{b}\left(v_{0}\right)$, we have $\mathrm{I}_{l}\left(v^{0}\right) \subset \mathrm{I}_{b}\left(v_{0}\right)$. More precisely, $\mathrm{I}_{l}\left(v^{0}\right)$ is an interval with $v^{0}$ and $v_{0}$ as extremities. Figures 4 and 5 present an example of the determination of intervals $\mathrm{I}_{b}\left(v_{0}\right)$ and $\mathrm{I}_{l}\left(v^{0}\right)$, and their sensitivity to the behavior of $\alpha$ in a neighborhood of $t=0$.

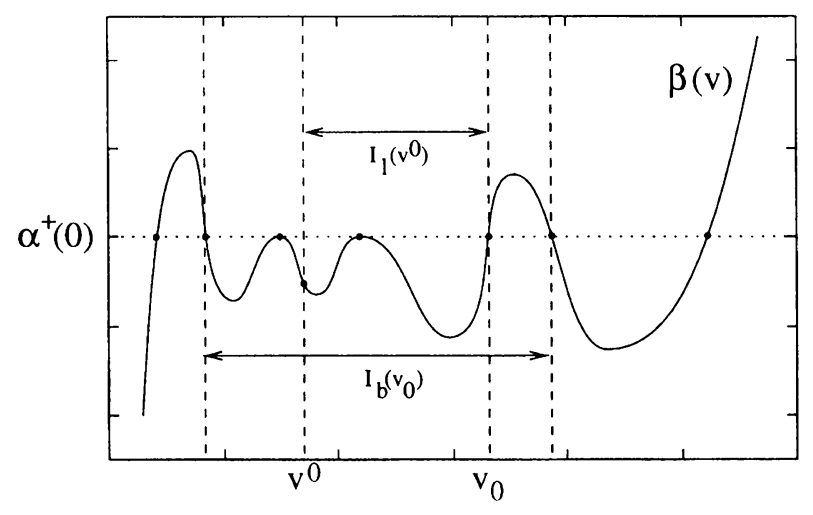

FIG. 4. Example of the determination of $v_{0}, \mathrm{I}_{l}\left(v^{0}\right)$ and $\mathrm{I}_{b}\left(v_{0}\right)$ with a strictly increasing $\alpha$

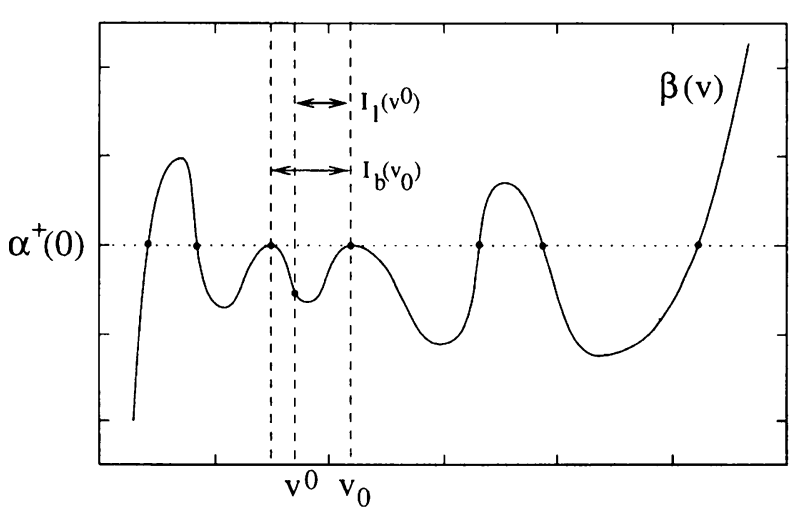

FIG. 5. Example of the determination of $v_{0}, \mathrm{I}_{l}\left(v^{0}\right)$ and $\mathrm{I}_{b}\left(v_{0}\right)$ with a decreasing $\alpha$

6.5. Behavior of the sequence of solutions in a neighborhood of $t=0$. In this subsection, we show that, whenever $\left(\alpha^{+}(0), v^{0}\right)$ is not an unstable point of $\beta$, the sequence $v_{\varepsilon}$ converges pointwise in a neighborhood of $t=0$. Actually, in this case $\mathrm{I}_{l}\left(v^{0}\right)$ contains a unique attractive point $v_{0}$, and by the definition of an attractive point, there exist a strictly increasing branch $\left[v_{1}, v_{2}\right],\left[\alpha_{1}, \alpha_{2}\right]$ of $\beta$ and $\zeta>0$ such that

$$
v_{0} \in\left[v_{1}, v_{2}\right], \quad \alpha^{+}(t) \in\left[\alpha_{1}, \alpha_{2}\right], \quad \forall t \in[0, \zeta] .
$$


That is why we first consider the case with $\beta$ strictly increasing. We can formulate the following results.

Proposition 7. With $\varepsilon>0, \alpha \in B V(0, T)$ and $\beta$ strictly increasing which satisfies Hypothesis 1, we have the following properties:

(1) If $v_{1}$ and $v_{2}$ are solutions of problem (15) with respective initial values $v_{1}^{0}$ and $v_{2}^{0}$, then the difference $\left|v_{1}(t)-v_{2}(t)\right|$ is decreasing on the interval $[0, T]$.

(2) Let $\alpha_{i}$ be a sequence of functions in $B V(0, T)$ that uniformly converges to a function $\alpha \in B V(0, T)$. Then the sequence $v_{\varepsilon i}$ of the solutions of problem (15) for the respective data $\alpha_{i}$ uniformly converges to $v_{\varepsilon}$, the solution of problem (15) for the data $\alpha$. Moreover, this convergence is uniform in $\varepsilon$.

Proof. Property 1: Both $v_{1}$ and $v_{2}$ are solutions of problem (15). Thus

$$
\varepsilon \frac{d}{d t} v_{1}(t)-\varepsilon \frac{d}{d t} v_{2}(t) \in \beta\left(v_{2}\right)-\beta\left(v_{1}\right), \quad \text { a.e. on }[0, T] \text {. }
$$

Also,

$$
\varepsilon\left(\frac{d}{d t} v_{1}(t)-\frac{d}{d t} v_{2}(t)\right)\left(v_{1}-v_{2}\right) \in\left(\beta\left(v_{2}\right)-\beta\left(v_{1}\right)\right)\left(v_{1}-v_{2}\right), \quad \text { a.e. on }[0, T],
$$

and because $\beta$ is increasing,

$$
\varepsilon \frac{d}{d t}\left(v_{1}-v_{2}\right)^{2} \leq 0, \quad \text { a.e. on }[0, T] .
$$

Property 2: The map $\beta$ has an increasing uni-valued continuous reciprocal function that we denote by $\beta^{-1}$. Let us set

$$
M=\sup _{\varepsilon>0, i \geq 0}\left\|v_{\varepsilon i}\right\|_{L^{\infty}(0, T)}, \quad A=\sup _{i \geq 0}\left\|\alpha_{i}\right\|_{L^{\infty}(0, T)} .
$$

We have $A<+\infty$ from the uniform convergence of the $\alpha_{i}$, and $M<+\infty$ because $M$ can be expressed directly as a function of $A$ (see proof of Proposition 2). The function $\beta^{-1}$ is continuous on $[-M,+M]$. Thus it is uniformly continuous and we can write

$$
\forall \gamma>0, \exists \eta_{\gamma}>0 ; \forall a_{1}, a_{2} \in[-A,+A],\left|a_{1}-a_{2}\right|<\eta_{\gamma} \Rightarrow\left|\beta^{-1}\left(a_{1}\right)-\beta^{-1}\left(a_{2}\right)\right|<\gamma .
$$

Using the increasing property of $\beta^{-1}$ we can rewrite this as follows:

$$
\forall \gamma>0, \exists \eta_{\gamma}>0 ; \forall a_{1}, a_{2} \in[-A,+A], a_{2}-a_{1} \leq \eta_{\gamma} \Rightarrow \beta^{-1}\left(a_{2}\right)-\beta^{-1}\left(a_{1}\right) \leq \gamma .
$$

Setting $w_{1}=\beta^{-1}\left(a_{1}\right)$ and $w_{2}=\beta^{-1}\left(a_{2}\right)$, we obtain

$$
\forall \gamma>0, \exists \eta_{\gamma}>0 ; \forall w_{1}, w_{2} \in[-M,+M], \min \left(\beta\left(w_{2}\right)-\beta\left(w_{1}\right)\right) \leq \eta_{\gamma} \Rightarrow w_{2}-w_{1} \leq \gamma,
$$

and also,

$$
\forall \gamma>0, \exists \eta_{\gamma}>0 ; \forall w_{1}, w_{2} \in[-M,+M], w_{2}-w_{1}>\gamma \Rightarrow \beta\left(w_{2}\right)-\beta\left(w_{1}\right)>\eta_{\gamma} .
$$

For any $\eta>0$ let us set

$$
\delta(\eta)=\inf \left\{\gamma>0: \text { assertion (17) is satisfied with } \eta_{\gamma}=\eta\right\} \text {. }
$$

It is clear that the value of $\delta(\eta)$ tends to zero when $\eta$ tends to zero. We prove the following property to end the demonstration:

$$
\left\|v_{\varepsilon i}-v_{\varepsilon}\right\|_{L^{\infty}(0, T)} \leq \delta\left(\left\|\alpha_{i}-\alpha\right\|_{L^{\infty}(0, T)}\right) .
$$


Assume that there exists $t_{1} \in[0, T]$ such that

$$
v_{\varepsilon i}\left(t_{1}\right)>v_{\varepsilon}\left(t_{1}\right)+\delta\left(\left\|\alpha_{i}-\alpha\right\|_{L^{\infty}(0, T)}\right) .
$$

Thanks to the continuity of $v_{\varepsilon i}$ and $v_{\varepsilon}$, there exists $\left.t_{0} \in\right] 0, t_{1}[$ such that

$$
v_{\varepsilon i}\left(t_{0}\right)=v_{\varepsilon}\left(t_{0}\right)+\delta\left(\left\|\alpha_{i}-\alpha\right\|_{L^{\infty}(0, T)}\right)
$$

and

$$
\left.\left.v_{\varepsilon i}(t)>v_{\varepsilon}(t)+\delta\left(\left\|\alpha_{i}-\alpha\right\|_{L^{\infty}(0, T)}\right), \quad \text { for } t \in\right] t_{0}, t_{1}\right] .
$$

Hence we also have

$$
\left.\left.\varepsilon \frac{d}{d t} v_{\varepsilon i}-\varepsilon \frac{d}{d t} v_{\varepsilon} \in \alpha_{i}(t)-\alpha(t)-\beta\left(v_{\varepsilon i}\right)+\beta\left(v_{\varepsilon}\right) \text {, a.e. on }\right] t_{0}, t_{1}\right] \text {. }
$$

Thus,

$$
\left.\left.\varepsilon \frac{d}{d t} v_{\varepsilon i}-\varepsilon \frac{d}{d t} v_{\varepsilon}<0 \text { a.e. on }\right] t_{0}, t_{1}\right]
$$

a contradiction. Similarly it is possible to establish that

$$
v_{\varepsilon i}(t) \geq v_{\varepsilon}(t)-\delta\left(\left\|\alpha_{i}-\alpha\right\|_{L^{\infty}(0, T)}\right), \quad \forall t \in[0, T],
$$

and the proof is completed.

It is now possible to formulate the following convergence result:

Proposition 8. With $\alpha \in B V(0, T)$ and $\beta$ strictly increasing verifying Hypothesis 1 , the sequence $v_{\varepsilon}$ of the solutions of problem (15) converges pointwise to a function $v_{0}$ when $\varepsilon$ tends to zero. Moreover, $v_{0}$ is left continuous, has a right limit everywhere on ] $0, T]$ and satisfies:

$$
\begin{aligned}
& v_{0}^{+}(t) \text { is an attractive point for all } t \in[0, T[ \\
& \left.\left.v_{0}(t) \text { is a backward attractive point for all } t \in\right] 0, T\right] .
\end{aligned}
$$

First, we establish the following intermediary result:

LEMma 5. The assertion of Proposition 8 is valid with $\alpha$ a step function.

Proof of the lemma. It is sufficient to prove the result with only one point of discontinuity. The proof can be obviously extended to a finite number of intervals. Let us take

$$
\alpha(t)=\left\{\begin{array}{l}
a_{0} \text { if } t \in\left[0, t_{0}\right), \\
a_{1} \text { if } t \in\left(t_{0}, T\right] .
\end{array}\right.
$$

The point $\left(\alpha^{+}(0), v^{0}\right)$ cannot be an unstable point. Thus, $\mathrm{I}_{l}\left(v^{0}\right)$ contains a unique attractive point $v_{0}$. Using Lemma 4 , for all $\delta>0$, there exists $\zeta_{2}>0$ such that for all $\left.\left.\zeta_{1} \in\right] 0, \zeta_{2}\right]$ there exists $\varepsilon_{0}>0$ with

$$
\left.\left.v_{\varepsilon}(t) \in\left[v_{0}-\delta, v_{0}+\delta\right], \forall t \in\left[\zeta_{1}, \zeta_{2}\right], \forall \varepsilon \in\right] 0, \varepsilon_{0}\right] .
$$

From property 1 of Proposition 7 the difference $\left|v_{\varepsilon}(t)-v_{0}\right|$ is decreasing on $\left[0, t_{0}\right]$. Thus,

$$
v_{\varepsilon}(t) \in\left[v_{0}-\delta, v_{0}+\delta\right], \forall t \in\left[\zeta_{1}, t_{0}\right],
$$


and so, the sequence $v_{\varepsilon}$ uniformly converges to the value $v_{0}$ on $\left[\zeta_{1}, a\right]$, for all $\left.\zeta_{1} \in\right] 0, t_{0}[$. On the interval $\left[t_{0}, T\right]$ we consider the following Cauchy problem:

$$
\left\{\begin{array}{l}
\varepsilon \frac{d}{d t} \tilde{v_{\varepsilon}}(t) \in \alpha(t)-\beta\left(\tilde{v_{\varepsilon}}(t)\right), \text { a.e. on }\left[t_{0}, T\right], \\
\tilde{v}_{\varepsilon}\left(t_{0}\right)=v_{0} .
\end{array}\right.
$$

Similarly, the sequence $\tilde{v}_{\varepsilon}$ converges uniformly on every interval $\left[t_{0}+\zeta_{1}, T\right]$ to a constant value $\tilde{v}_{0}$. Moreover, at $t=t_{0}$, the sequence $v_{\varepsilon}$ converges to the value $v_{0}$. Property 1 of Proposition 7 implies

$$
\left|\tilde{v_{\varepsilon}}(t)-v_{\varepsilon}(t)\right|<\left|v_{0}-v_{\varepsilon}\left(t_{0}\right)\right| \forall t \in\left[t_{0}, T\right],
$$

which means that the sequence $v_{\varepsilon}$ uniformly converges to the constant value $\tilde{v}_{0}$ on the whole interval $\left[t_{0}+\zeta_{1}, T\right]$. The additional properties of $v_{0}$ are obviously verified.

Proof of Proposition 8. Since the function $\alpha$ has bounded variation, it can be uniformly approximated by a sequence of step functions $\alpha_{i}$. Let $v_{\varepsilon i}$ be the solutions of problem (15) for the respective data $\alpha_{i}$ and $v_{\varepsilon}$ the solution of problem (15) for the data $\alpha$. Using Lemma 5 , there exist functions $v_{0 i}$ such that, for each $i$, the sequence $v_{\varepsilon i}$ converges pointwise to $v_{0 i}$ when $\varepsilon$ tends to zero, with $\alpha_{i}^{-}(t) \in \beta\left(v_{0 i}(t)\right)$ on $\left.] 0, T\right]$. Property 2 of Proposition 7 implies that $v_{\varepsilon i}$ uniformly converges on $[0, T]$ to $v_{\varepsilon}$ when $i$ tends to $+\infty$ and uniformly in $\varepsilon$. Thus, for $t \in] 0, T]$ we have

$$
\forall \delta>0, \exists \tilde{N}>0, \exists \varepsilon(\tilde{N})>0 ; \varepsilon_{1}, \varepsilon_{2}<\varepsilon(\tilde{N}) \Rightarrow\left\{\begin{array}{l}
\left|v_{\varepsilon_{1} \tilde{N}}(t)-v_{\varepsilon_{2} \tilde{N}}(t)\right|<\delta / 3 \\
\left|v_{\varepsilon_{2} \tilde{N}}(t)-v_{\varepsilon_{2}}(t)\right|<\delta / 3 \\
\left|v_{\varepsilon_{1} \tilde{N}}(t)-v_{\varepsilon_{1}}(t)\right|<\delta / 3
\end{array}\right.
$$

hence,

$$
\forall \delta>0, \exists \tilde{N}>0, \exists \varepsilon(\tilde{N})>0 ; \varepsilon_{1}, \varepsilon_{2}<\varepsilon(\tilde{N}) \Rightarrow\left|v_{\varepsilon_{1}}(t)-v_{\varepsilon_{2}}(t)\right|<\delta .
$$

So, the sequence $v_{\varepsilon}(t)$ is a Cauchy sequence when $\varepsilon$ tends to zero. Thus it converges pointwise to a function $v_{0}(t)$. Moreover, $v_{0}$ is the uniform limit of the functions $v_{0 i}$ because, using the different convergences, we can write

$$
\begin{aligned}
\forall \delta>0, \exists \tilde{N}>0 ; \forall i \geq \tilde{N}, \forall t \in[0, T], \exists \varepsilon(i, t)>0, & \varepsilon<\varepsilon(i, t) \\
& \Rightarrow\left\{\begin{array}{r}
\left|v_{\varepsilon i}(t)-v_{0 i}(t)\right|<\delta / 3, \\
\left|v_{\varepsilon}(t)-v_{0}(t)\right|<\delta / 3, \\
\left|v_{\varepsilon i}(t)-v_{\varepsilon}(t)\right|<\delta / 3 .
\end{array}\right.
\end{aligned}
$$

Thus,

$$
\forall \delta>0, \exists \tilde{N}>0 ; \forall i \geq \tilde{N}, \forall t \in[0, T],\left|v_{0}(t)-v_{0 i}(t)\right|<\delta .
$$

In order to establish the left continuity of $v_{0}$, we can write

$$
v_{0}(t)=\lim _{i \rightarrow \infty} v_{0 i}(t)=\lim _{i \rightarrow \infty} \beta^{-1}\left(\alpha_{i}^{-}(t)\right)=\beta^{-1}\left(\lim _{i \rightarrow \infty} \alpha_{i}^{-}(t)\right) .
$$

Using the uniform convergence of the $\alpha_{i}$ to $\alpha$ we have

$$
\lim _{i \rightarrow \infty} \alpha_{i}^{-}(t)=\alpha^{-}(t)
$$


This proves that $v_{0}(t)$ is a backward attractive point. In the same manner, for the right limit we have

$$
v_{0}^{+}(t)=\lim _{i \rightarrow \infty} v_{0 i}^{+}(t)=\lim _{i \rightarrow \infty} \beta^{-1}\left(\alpha_{i}^{+}(t)\right)=\beta^{-1}\left(\alpha^{+}(t)\right) .
$$

This proves that $v_{0}^{+}(t)$ is an attractive point.

We can now describe the behavior of the sequence $v_{\varepsilon}$ in a neighborhood of $t=0$ :

Proposition 9. With $\alpha \in B V(0, T), \beta$ satisfying Hypotheses 1 and 2 and the point $\left(\alpha^{+}(0), v^{0}\right)$ not being an unstable point of $\beta$, there exist a strictly increasing branch $\left[v_{1}, v_{2}\right],\left[\alpha_{1}, \alpha_{2}\right]$ of $\beta$ and $\zeta>0$ such that the sequence $v_{\varepsilon}$ of the solutions of problem (15) converges pointwise to a function $v_{0}$ on $[0, \zeta]$. Moreover, $v_{0}$ is left continuous, has a right limit everywhere on $] 0, \zeta]$ and satisfies:

$v_{0}^{+}(t)$ is an attractive point for all $t \in[0, \zeta[$,

$v_{0}(t)$ is a backward attractive point for all $\left.\left.t \in\right] 0, \zeta\right]$.

Proof. Because the point $\left(\alpha^{+}(0), v^{0}\right)$ is not an unstable point, $\mathrm{I}_{l}\left(v^{0}\right)$ contains a unique attractive point $v_{00}$. Using the definition of an attractive point there exist a strictly increasing branch $\left[v_{1}, v_{2}\right],\left[\alpha_{1}, \alpha_{2}\right]$ of $\beta$ and $\zeta>0$ such that

$$
v_{00} \in\left[v_{1}, v_{2}\right], \quad \alpha^{+}(t) \in\left[\alpha_{1}, \alpha_{2}\right], \quad \forall t \in[0, \zeta[.
$$

On the interval $[0, \zeta]$ we consider the following Cauchy problem:

$$
\left\{\begin{array}{l}
\varepsilon \frac{d}{d t} \tilde{v_{\varepsilon}}(t) \in \alpha(t)-\beta\left(\tilde{v}_{\varepsilon}(t)\right), \text { a.e. on }[0, \zeta], \\
\tilde{v}_{\varepsilon}\left(t_{0}\right)=v_{00} .
\end{array}\right.
$$

We can apply Proposition 8 to this problem because we have, from Proposition $1, \tilde{v_{\varepsilon}}(t) \in$ $\left[v_{1}, v_{2}\right]$ for all $t \in[0, \zeta]$ and for all $\varepsilon>0$. Thus, the sequence $\tilde{v}_{\varepsilon}$ converges pointwise to a function $\tilde{v}_{0}$ on $[0, \zeta]$ when $\varepsilon$ tends to zero. Moreover, $v_{0}$ has the expected properties. We still have to prove that the sequence $v_{\varepsilon}$ converges pointwise to $v_{0}$ too.

Assume $v_{00}>v^{0}$ (for $v_{00}=v^{0}$ we can apply directly Proposition 8 and for $v_{00}<v^{0}$ the proof is similar). The strictly increasing branch $\left[v_{1}, v_{2}\right],\left[\alpha_{1}, \alpha_{2}\right]$ is included in a strictly increasing branch $\left[v_{i}^{*}, v_{i+1}^{*}\right],\left[\alpha_{i}^{*}, \alpha_{i+1}^{*}\right]$ of $\beta$ with $\left(\alpha_{i}^{*}, v_{i}^{*}\right)$ a critical minimum point. Necessarily $v_{00}>v_{i}^{*}$ because otherwise $\mathrm{I}_{l}\left(v^{0}\right)=\left[v^{0}, v_{i}^{*}\right]$ with a $\left.v_{1} \in\right] v^{0}, v_{i}^{*}[$ such that $\beta(v)>\alpha^{+}(0)$, which is impossible if we consider Lemma 2. From Lemma 4, for all $\delta>0$, there exists $\zeta_{2}>0$ such that for all $\left.\left.\zeta_{1} \in\right] 0, \zeta_{2}\right]$ there exists $\varepsilon_{0}>0$ with

$$
\left.\left.v_{\varepsilon}(t) \in\left[v_{00}-\delta, v_{00}+\delta\right], \forall t \in\left[\zeta_{1}, \zeta_{2}\right], \forall \varepsilon \in\right] 0, \varepsilon_{0}\right] \text {. }
$$

For $\delta<v_{00}-v_{i}^{*}$, Property 1 of Proposition 7 implies that $\left|v_{\varepsilon}(t)-\tilde{v}_{\varepsilon}(t)\right|$ is decreasing on $\left[\zeta_{1}, \zeta\right]$. Thus $v_{\varepsilon}-\tilde{v}_{\varepsilon}(t)$ converges uniformly to zero on all intervals $\left[\zeta_{1}, \zeta\right]$ with $\zeta_{1}>0$.

\section{General convergence result.}

Theorem 1. Assume that $\alpha \in B V(0, T), \beta$ satisfies Hypotheses 1 and 2 and the point $\left(\alpha^{+}(0), v^{0}\right)$ is not an unstable point of $\beta$. Then, the sequence $v_{\varepsilon}$ of the solutions of 
problem (15) converges pointwise to a function $v_{0}$ when $\varepsilon$ tends to zero. Moreover, $v_{0}$ is left continuous, has a right limit everywhere on $] 0, T]$ and satisfies:

$$
\begin{aligned}
& v_{0}^{+}(t) \text { is an attractive point for all } t \in[0, T[ \\
& \left.\left.v_{0}(t) \text { is a backward attractive point for all } t \in\right] 0, T\right] .
\end{aligned}
$$

Proof. From Proposition 9 we know that Theorem 1 holds in a neighborhood of $t=0$. We saw in the proof of this proposition that there exists a strictly increasing branch $\left[v_{i}^{*}, v_{i+1}^{*}\right],\left[\alpha_{i}^{*}, \alpha_{i+1}^{*}\right]$ of $\beta$ with $\alpha^{+}(0) \in\left[\alpha_{i}^{*}, \alpha_{i+1}^{*}\right]$ such that, if we set

$$
t_{0}=\sup \left\{t: \alpha^{+}(\tau) \in\left[\alpha_{i}^{*}, \alpha_{i+1}^{*}\right] \forall \tau \in[0, t[\},\right.
$$

then, the sequence $v_{\varepsilon}$ converges pointwise on $\left[0, t_{0}\right]$ to a function $v_{0}$ that has the expected properties. If now we consider the following Cauchy problem:

$$
\left\{\begin{array}{l}
\varepsilon \frac{d}{d t} \tilde{v}_{\varepsilon}(t) \in \alpha(t)-\beta\left(\tilde{v}_{\varepsilon}(t)\right), \text { a.e. on }\left[t_{0}, T\right], \\
\tilde{v}_{\varepsilon}\left(t_{0}\right)=v_{0}\left(t_{0}\right),
\end{array}\right.
$$

then we can apply Proposition 9 to this problem. So there exists a strictly increasing branch $\left[v_{j}^{*}, v_{j+1}^{*}\right],\left[\alpha_{j}^{*}, \alpha_{j+1}^{*}\right]$ of $\beta$ which is different from the previous one, with $\alpha^{+}\left(t_{0}\right) \in$ $\left[\alpha_{j}^{*}, \alpha_{j+1}^{*}\right]$ such that if we set

$$
t_{1}=\sup \left\{t: \alpha^{+}(\tau) \in\left[\alpha_{j}^{*}, \alpha_{j+1}^{*}\right] \forall \tau \in\left[t_{0}, t[\},\right.\right.
$$

then the sequence $\tilde{v}_{\varepsilon}$ converges pointwise on $\left[t_{0}, t_{1}\right]$ to a function $\tilde{v}_{0}$ that has the expected properties.

We denote by $\tilde{\mathrm{I}}_{l}\left(v_{0}\left(t_{0}\right)\right)$ and $\tilde{\mathrm{I}}_{b}\left(\tilde{v}_{0}^{+}\left(t_{0}\right)\right)$, respectively, the interval of all limit points and the region of attraction of the boundary layer for problem (18).

Because $v_{0}\left(t_{0}\right) \in\left[v_{i}^{*}, v_{i+1}^{*}\right]$ and $\tilde{v}_{0}^{+}\left(t_{0}\right) \in\left[v_{j}^{*}, v_{j+1}^{*}\right]$ we have necessarily $v_{0}\left(t_{0}\right) \neq \tilde{v}_{0}^{+}\left(t_{0}\right)$. Thus $v_{0}\left(t_{0}\right)$ is neither an attractive point nor an unstable point. So $v_{0}\left(t_{0}\right)$ belongs to the interior of $\tilde{\mathrm{I}}_{b}\left(\tilde{v}_{0}^{+}\left(t_{0}\right)\right)$, because an extremity of $\tilde{\mathrm{I}}_{b}\left(\tilde{v}_{0}^{+}\left(t_{0}\right)\right)$ is either an unstable point or an attractive critical point. Thus for $\delta>0$ sufficiently small we have

$$
\left[v_{0}\left(t_{0}\right)-\delta, v_{0}\left(t_{0}\right)+\delta\right] \subset \tilde{\mathrm{I}}_{b}\left(\tilde{v}_{0}^{+}\left(t_{0}\right)\right)
$$

Because $v_{\varepsilon}\left(t_{0}\right)$ converges to $v_{0}\left(t_{0}\right)$ when $\varepsilon$ tends to zero, there exists $\varepsilon_{0}>0$ such that $v_{\varepsilon}\left(t_{0}\right) \in\left[v_{0}\left(t_{0}\right)-\delta, v_{0}\left(t_{0}\right)+\delta\right]$ for all $\left.\left.\varepsilon \in\right] 0, \varepsilon_{0}\right]$.

Let $v_{\varepsilon}{ }^{a}$ be the solution of problem (18) with initial value $v_{\varepsilon}{ }^{a}\left(t_{0}\right)=v_{0}\left(t_{0}\right)-\delta$ and let $v_{\varepsilon}^{b}$ be the solution of problem (18) with initial value $v_{\varepsilon}^{b}\left(t_{0}\right)=v_{0}\left(t_{0}\right)+\delta$. On the interval $\left[t_{0}, t_{1}\right]$ we have $v_{\varepsilon}(t) \in\left[v_{\varepsilon}^{a}(t), v_{\varepsilon}^{b}(t)\right]$. From Proposition 9 , the two sequences $v_{\varepsilon}^{a}$ and $v_{\varepsilon}^{b}$ converge to $\tilde{v}_{0}$ when $\varepsilon$ tends to zero; thus $v_{\varepsilon}$ converges to $\tilde{v}_{0}$ too.

The previous reasoning can be obviously extended to a finite number of branch changes. To conclude the claim, it is sufficient to prove that there is necessarily only a finite number of branch changes on the interval $[0, T]$.

Assume that the point $\left(\alpha^{+}(0), v_{0}\right)$ belongs to a strictly increasing branch $\left[\alpha_{i_{0}}^{*}, \alpha_{i_{0}+1}^{*}\right]$, $\left[v_{i_{0}}^{*}, v_{i_{0}+1}^{*}\right]$ of $\beta$. The first transition between two increasing branches holds at a time $t=t_{1}$ only if either $\alpha^{+}\left(t_{1}\right) \geq \alpha_{i_{0}+1}^{*}$ or $\alpha^{+}\left(t_{1}\right) \leq \alpha_{i_{0}}^{*}$ and toward another strictly increasing branch $\left[\alpha_{i_{1}}^{*}, \alpha_{i_{1}+1}^{*}\right],\left[v_{i_{1}}^{*}, v_{i_{1}+1}^{*}\right]$ of $\beta$. In the same way, the second transition holds at a 
time $t=t_{2}$ only if either $\alpha^{+}\left(t_{2}\right) \geq \alpha_{i_{1}+1}^{*}$ or $\alpha^{+}\left(t_{2}\right) \leq \alpha_{i_{1}}^{*}$ and toward a strictly increasing branch $\left[\alpha_{i_{2}}^{*}, \alpha_{i_{2}+1}^{*}\right],\left[v_{i_{2}}^{*}, v_{i_{2}+1}^{*}\right]$ of $\beta$. We can conclude

$$
\left|\alpha^{+}(0)-\alpha^{+}\left(t_{1}\right)\right|+\left|\alpha^{+}\left(t_{1}\right)-\alpha^{+}\left(t_{2}\right)\right|>\min _{1 \leq i \leq 2 N-1}\left|\alpha_{i}^{*}-\alpha_{i+1}^{*}\right| .
$$

More generally, if $t_{i}$, for $1 \leq i \leq M$, are the transition times between two strictly increasing branches of $\beta$, then

$$
\sum_{i=1}^{M-1}\left|\alpha^{+}\left(t_{i}\right)-\alpha^{+}\left(t_{i+1}\right)\right| \geq \frac{M}{2} \min _{1 \leq i \leq 2 N-1}\left|\alpha_{i}^{*}-\alpha_{i+1}^{*}\right| .
$$

If we denote by $V_{0}^{T} \alpha^{+}$the total variation of $\alpha^{+}$, which is finite because $\alpha \in B V(0, T)$, we have

$$
\sum_{i=1}^{M-1}\left|\alpha^{+}\left(t_{i}\right)-\alpha^{+}\left(t_{i+1}\right)\right| \leq V_{0}^{T} \alpha^{+}
$$

and thus

$$
M \leq 2 * \frac{V_{0}^{T} \alpha^{+}}{\min _{1 \leq i \leq 2 N-1}\left|\alpha_{i}^{*}-\alpha_{i+1}^{*}\right|},
$$

and the number of transition times is necessarily finite.

8. Connection with the perfect delay criterion. The expressions (10) and (11) of the perfect delay criterion only make sense for sufficiently smooth solutions. Assuming that $\beta$ satisfies Hypotheses 1 and 2, we will compare the limit solution of the perturbed problem (15) and the solution selected by the perfect delay criterion in some cases that show global behavior.

a) Strictly increasing branch of $\beta$ and $\alpha$ continuous. Assume that $\alpha$ is continuous and that the solution remains on a strictly increasing branch $\left[\alpha_{1}, \alpha_{2}\right],\left[v_{1}, v_{2}\right]$ of $\beta$. Proposition 8 implies that the limit solution $v_{0}(t)$ of the perturbed problem satisfies $v_{0}(t) \in\left[v_{1}, v_{2}\right]$. Moreover, the map $\beta$ has a continuous inverse function on the strictly increasing branch. It implies that $v_{0}$ is continuous. Thus in this case the solution $v_{0}$ respects the perfect delay criterion.

b) Strictly increasing branch of $\beta$ and $\alpha$ discontinuous. If now $\alpha$ has a point of discontinuity $\alpha^{-}\left(t_{1}\right) \neq \alpha^{+}\left(t_{1}\right)$, the limit solution that still satisfies $v_{0}(t) \in\left[v_{1}, v_{2}\right]$ is discontinuous at $t=t_{1}$. The expression (10) of the perfect delay criterion gives no information on which jump to choose. Each value $v_{0}^{+}\left(t_{1}\right) \in \beta^{-1}\left(\alpha^{+}\left(t_{1}\right)\right)$ is acceptable. This expression does not always allow us to select a unique solution. Expression (11) of the criterion tells us that the smallest jump has to be chosen. This additional information is not always sufficient because two jumps can have the same length. Moreover, the choice of the smallest jump is not always compatible with the limit solution $v_{0}$.

c) Critical maximum point of $\beta$ and $\alpha$ continuous and increasing. Now we assume that $\alpha$ is continuous and strictly increasing, that the solution remains on a strictly increasing branch of $\beta$, and that for a given time $t=t_{1}$ the function $\alpha\left(t_{1}\right)$ reaches a critical maximum point $\left(v_{1}, \alpha\left(t_{1}\right)\right)$ of $\beta$. There does not exist a continuous solution of the inclusion (6). As in the previous case, the expression (10) of the perfect delay criterion does not indicate 
which jump is to be chosen. Expression (11) indicates that the smallest jump has to be chosen. As in the previous case, this choice is not always compatible with the limit solution $v_{0}$.

As a conclusion, for smooth enough solutions, when the expression (10) selects a unique solution of (6), this is the same as the limit solution of Theorem 1. Even though expression (11) gives a little more information on the jumps, this information is not always compatible with the limit solution $v_{0}$ and when it is not the case, the choice of the smallest jump is not clearly justified.

\section{REFERENCES}

[1] W. R. Brace and J. D. Byerlee, Stick-slip as a mechanism for earthquakes, Science 153, 990-992 (1966)

[2] M. Campillo, I. R. Ionescu, J.-C. Paumier, and Y. Renard, On the dynamic sliding with friction of a rigid block and of an infinite elastic slab, Physics of the Earth and Planetary Interiors 96, 15-23 (1996)

[3] K. Deimling, Multivalued Differential Equations, Walter de Gruyter, Berlin, 1992

[4] L. C. Evans and R. F. Gariepy, Measure theory and fine properties of functions, CRC Press, 1992

[5] C. Gao and D. Kuhlmann-Wilsdorf, On stick-slip and velocity dependence of friction at low speeds, ASME Journal of Tribology 112, 355-360 (1990)

[6] I. R. Ionescu and J.-C. Paumier, On the contact problem with slip rate dependent friction in elastodynamics, European J. Mech. A Solids 13, 555-568 (1994)

[7] A. I. Leonov and A. Srinivasan, Self-oscillations of an elastic plate sliding over a smooth surface, Internat. J. Engrg. Sci. 31, 453-473 (1993)

[8] E. Rabinowicz, Friction and Wear, Study of the Stick-slip Process, Davies, Elsevier, New York, 1949, pp. 149-164

[9] E. Rabinowicz, The intrinsic variables affecting the stick-slip process, Proceedings of the Royal Physical Society 71, 668-675 (1958)

[10] Y. Renard, Modélisation des instabilités liées au frottement sec des solides élastiques, aspects théoriques et numériques, thèse de doctorat, LMC-IMAG Grenoble (1998)

[11] W. Rudin, Analyse réelle et complexe, Masson, Paris, 1992

[12] D. Tabor, Friction: The present state of our understanding, J. of Lubrication Technology 103, 169-179 (1981)

[13] A. N. Tikhonov, A. B. Vasiléva, and A. G. Sveshnikov, Differential Equations, Chapter VII, Asymptotics of solutions of differential equations with respect to a small parameter, Springer-Verlag, New York, 1980, pp. 81-213 